\title{
A modularity analysis helps improving the structure of the International Code of Zoological Nomenclature
}

\author{
Evangelos Vlachos ${ }^{\text {Corresp. } 1}$ \\ ${ }^{1}$ Laboratory of Vertebrate Paleontology, CONICET and Museo Paleontologico Egidio Feruglio, Trelew, Chubut, Argentina, Trelew, Chubut, Argentina \\ Corresponding Author: Evangelos Vlachos \\ Email address: evlacho@mef.org.ar
}

Background. In a recent work I transformed a complex and integrated text like the International Code of Zoological Nomenclature into a network of interconnected parts of text. This new approach allowed understanding that a continuous body of text cannot accurately reflect the true structure of the Code, and provided a scientific methodology to identify a priori parts that could be affected by future revisions. In this next step, I investigate further the structure of the Code, seeking to use the network in order to identify the various conceptual communities grouping the various articles and other text items of the Code.

Methods. Using the first version of the network of the Code, I perform a comprehensive modularity analysis in two rounds: the first round aims to identify the fewest and largest communities or modules for the entire network, whereas the second round identifies the sub-modules within each larger module. The potential conflicts between the current structure of the Code and the module composition are evaluated with a parcellation analysis.

Results. The optimal modularity search identified 10 different modules in the entire network of varying size (ranging from 75 to 200 nodes). Each module can be further divided into smaller modules, that alltogether allow describing the 65 conceptual groups of text items in the Code. Parcellation analysis revealed that two-thirds of the current chapters of the Code are in excellent or good accordance with the recovered conceptual modules, whereas the current composition of six chapters is in serious conflict with the conceptual structure of the Code.

Discussion. Judging only the composition and not the order of appearance of the Articles in the Chapters of the Code, I show that in many cases the current structure of the Code is found to correspond quite well to the concepts presented therein. The most important conflict is found on the provisions related to the various groups of names governed by the Code: family-, genus-, and species-group names. Currently, these provisions are spread out in different Articles in different Chapters, along the entire length of the Code. The modularity analysis suggests that re-organizing the Code in chapters that will deal with all aspects related to a given group (e.g., chapters including information on name formation, availability, typification, and validity for a given group), could potentially improve reader experience and, consequently, the applicability of the Code. 
1 A modularity analysis helps improving the structure of

2 the International Code of Zoological Nomenclature

3

4 Evangelos Vlachos

5

6 CONICET - Museo Paleontológico Egidio Feruglio, Trelew, Chubut, Argentina

7

8 Corresponding Author:

9 Evangelos Vlachos

10 Av. Fontana 140, Trelew, Chubut, 9100, Argentina

11 Email address: evlacho@,mef.org.ar 


\section{Abstract}

13 Background. In a recent work I transformed a complex and integrated text like the International

14 Code of Zoological Nomenclature into a network of interconnected parts of text. This new

15 approach allowed understanding that a continuous body of text cannot accurately reflect the true

16 structure of the Code, and provided a scientific methodology to identify a priori parts that could

17 be affected by future revisions. In this next step, I investigate further the structure of the Code,

18 seeking to use the network in order to identify the various conceptual communities grouping the

19 various articles and other text items of the Code.

20 Methods. Using the first version of the network of the Code, I perform a comprehensive

21 modularity analysis in two rounds: the first round aims to identify the fewest and largest

22 communities or modules for the entire network, whereas the second round identifies the sub-

23 modules within each larger module. The potential conflicts between the current structure of the

24 Code and the module composition are evaluated with a parcellation analysis.

25 Results. The optimal modularity search identified 10 different modules in the entire network of

26 varying size (ranging from 75 to 200 nodes). Each module can be further divided into smaller

27 modules, that all-together allow describing the 65 conceptual groups of text items in the Code.

28 Parcellation analysis revealed that two-thirds of the current chapters of the Code are in excellent

29 or good accordance with the recovered conceptual modules, whereas the current composition of

30 six chapters is in serious conflict with the conceptual structure of the Code.

31 Discussion. Judging only the composition and not the order of appearance of the Articles in the

32 Chapters of the Code, I show that in many cases the current structure of the Code is found to

33 correspond quite well to the concepts presented therein. The most important conflict is found on

34 the provisions related to the various groups of names governed by the Code: family-, genus-, and 
35 species-group names. Currently, these provisions are spread out in different Articles in different

36 Chapters, along the entire length of the Code. The modularity analysis suggests that re-

37 organizing the Code in chapters that will deal with all aspects related to a given group (e.g.,

38 chapters including information on name formation, availability, typification, and validity for a 39 given group), could potentially improve reader experience and, consequently, the applicability of 40 the Code.

\section{Introduction}

43 Zoological Nomenclature, as defined by the International Code of Zoological Nomenclature

44 (henceforth: the Code) is governed by the International Commission on Zoological

45 Nomenclature (henceforth: the Commission) (ICZN 1999). Recently, I managed to combine

46 Zoological Nomenclature with recent advances in network theory, and create a new perspective

47 for the Code (Vlachos 2019). I successfully transformed the Code into a network — called

48 NETICON ZOOLOGICON — and started to understand how this scientific language functions. I

49 showed how a network framework for the Code helps predicting parts that need improvement

50 and parts that might be affected by upcoming revisions. This new framework can also allow

51 comparing different Codes, both those that are truly different to each other (e.g., the Zoological

52 and the Botanical Codes) or different editions of the same Code. The NETICON can also be used

53 to present the Code in a different way, improving both reader experience and usage, as well as

54 enhancing the teaching potential. This last point is fully investigated herein, with a modularity

55 analysis of the NETICON.

56 Any network of interconnected nodes has a structure, as some of the nodes are more

57 closely connected to each other than the rest of the network, forming clusters or communities 
58 called modules. The discovery of these modules is a principal objective for network science. In

59 this case, the recognition of several modules in the Code could reveal the true hierarchical

60 structure of the Code, and would allow to formulate hypotheses for future improvements and

61 changes.

62

63 Methods

64 The starting point for this analysis is NETICON ZOOLOGICON v. 1.0, the network constructed and 65 presented in Vlachos (2019); see therein for details regarding the network itself. The modularity 66 analysis is based on the built-in Blondel et al. (2008) algorithm, that follows an heuristic

67 approach, performed in the free software GEPHI 0.9.2 (Bastian et al. 2009). Starting from a

68 random point in the network, the algorithm heuristically tries to identify communities based on a

69 pre-defined resolution scale; herein, the maximum resolution (1.0) was selected, to allow finding

70 the largest communities or modules. Because of its heuristic approach, each run of the algorithm

71 may produce slightly different results in the composition of modules. This has been discussed

72 extensively in the literature, and methods have been proposed to decide the minimum number of

73 runs necessary to get the best results, which depends on the total number of nodes in the network

74 (Calatayud et al. 2019 and references therein). Blondel's (2008) algorithm is not affected from

75 the starting point and the final results show a variation in the scale of $10^{-2}$. The numbering of the

76 modules is random and depends on the random starting point of the modularity algorithm.

77 The modularity analysis made herein comprises two rounds. In the first round, the entire

78 network is analysed, running the algorithm with 1.0 resolution for 50 times, keeping the solution

79 with the maximum $\mathrm{Q}$. The second round is made on each of the large modules recovered during

80 the first round and filtered as separate modules, with another 25 runs of the algorithm with the 
81 same settings and always maximizing the value Q. The scope behind this two-round search is to

82 create an hierarchical structure of the conceptual communities of the Code. It must be noted here

83 that the current, de facto, hierarchical structure of the Code - the order and composition of

84 Chapters and their containing Articles — is also coded in the NETICON, however with edges of a

85 minimum weight compared to the conceptual ones (see Vlachos 2019 for more information). The

86 used algorithm takes into account both the weight and the direction of the edges. The decision to

87 include in the modularity analysis these structural connections is made because I would like to

88 propose the most conservative changes in the de facto structure of the Code. Another point that

89 should be noted here is that the composition of the recovered modules is not to be taken literally,

90 and especially for the nodes placed in the periphery of each module. These nodes are, actually, in

91 the contact zone between two or more modules and - especially in the cases where these nodes

92 are equally connected with both modules — most times their placement in one or the other

93 module changed during each run. Therefore, some of the "solitary" items that might appear in

94 some modules could actually equally be placed in another module. What I am interested here is

95 the main and general composition of each module.

96 The comparison between the recovered modules is made with a modified Parcellation

97 Index (PI) per Chapter and Article, originally developed for Anatomical Networks in Esteve-

98 Altava et al. (2019). Once the analyses are made, in each Chapter/Article I count the number of

99 items include in each recovered module. For a network of $\mathrm{N}$ nodes and 3 modules, for example,

100 the PI would be calculated as such: $\mathrm{PI}=1-\left[\left(\mathrm{N}_{1} / \mathrm{N}\right)^{2}+\left(\mathrm{N}_{2} / \mathrm{N}\right)^{2}+\left(\mathrm{N}_{3} / \mathrm{N}\right)^{2}\right]($ Esteve-Altava et al.

101 2019); this metric takes into account not only how many modules are present, but also the

102 number of Nodes in each module. A Chapter/Article that has all its items recovered in a single

103 module would have a $\mathrm{PI}=0$, whereas a Chapter/Article that an equal number of nodes placed in 
104 all recovered modules would have a PI=1. Ideally, the de facto structure should match the 105 conceptual structure, i.e., each chapter should be included in one module and thus having a PI 106 close or equal to zero. On the contrary, if a chapter is included in many different modules, it 107 means that its composition is in conflict with the conceptual structure of the Code. The network 108 allows describing precisely this issue, by calculating the Chapter and Article individual 109 parcellation index. If all the parts of one Chapter are included in a single module, then its 110 parcellation index is 0 ; this means that the de facto structure of these Chapters is in harmony 111 with the conceptual structure of the Code. This technique helps evaluating the structure of the

112 various Chapters of the Code. We could divide, arbitrarily, the parcellation spectrum in four 113 equal areas: those Chapters with PI between $0-0,250$ have excellent structure, taking into 114 account the issue with the solitary items explained above, that could slightly increase the PI;

115 Chapters with PI between $0,250-0,500$ have good structure, as the great majority of their items is 116 included in one Module; Chapters with PI between 0,500-0,750 have problematic structure, as 117 their items are spread across many Modules; Chapters with PI between 0,750-1,000 have bad 118 structure, being parcellated across nearly all modules and with several items included in each 119 one. This evaluation can also help improving the reader experience of the Code. A Chapter with 120 low PI is a Chapter that can be read separately, whereas a Chapter with high PI requires reading 121 it within the entire context of the Code.

122

\section{Results}

124 The optimal modularity search (run \#23/50) discovered 10 different modules (module 0 125 module 9; keeping the original numbering of the software), with a $\mathrm{Q}$ of 0,586 (standard 126 deviation $=0,004$ among the 50 runs, which is within the range reported by Blondel et al. 2008) 
127 and a high parcellation index of 0,889 (Table 1 and Online Supplementary Information). The

128 modularity analysis reveals that the Code has a highly modular structure, meaning that it could

129 be divided in at least 10 distinct and large groups that contain text items that are more closely

130 connected to each other compared to the rest of the Code. This means that exist at least 10

131 different conceptual and general themes developed across the text of the Code. The high general

132 parcellation index corroborates the highly modular structure of the Code. This number is to be

133 interpreted as not only having many modules, but mainly that each one of these modules is

134 important for the entire structure.

135

136

Module 0 contains 181 Nodes and 833 Edges, representing 13,13\% of the Code; it is the

137

138

139

140

141

142

143

144

145

146

147

148

149

third-largest module in the network (Figure 1). Module 0 mostly contains items from Chapter 1 (Zoological Nomenclature; most items of Art. 1, and the entire Arts. 2, 3), Chapter 3 (Criteria of

Publication; nearly all items, excluding Rec. 8B which forms part of Module 8), some items from Chapter 4 (Criteria of Availability; parts of Art. 10 and Art. 17), most of Chapter 5 (Date of

Publication; the entire Art. 21, and some parts of Art. 22), as well as some parts from Chapters 6 (Rec. 24A), 8 (Art. 40.2.1, and Rec. 40A), 11 (Recs. 50B, 50C, 51F), 12 (Art. 54.1), and 17 (Art. 78.2.4). The most important Glossary terms included in this Module are: publish, date of publication, scientific name, publication, nomenclatural act, and zoological nomenclature. This Module also contains the Code of Ethics (Appendix A) and General Recommendations (Appendix B). Therefore, Module 0 covers some Generalities and introductory information of the Code, as well as what constitutes published and unpublished work. A secondary modularity analysis allows understanding better the conceptual structure of Module 0 . It consists of nine sub-modules, which deal with the following topics (Figure 2): sub-module 0.0: Introduction and 
150 Generalities and what is excluded from the Code and articles on the availability of excluded

151 names (Arts. 1-3, Art. 10.5, Arts. 17.1-3, Rec. 50C, Rec. 51F, Art. 54.1); sub-module 0.1: what

152 constitutes published work, excluding electronic publication (almost the entire Art. 8, excl. the

153 sub-articles of 8.4 and 8.5); sub-module 0.2 : what does not constitute published work (Art. 9);

154 sub-module 0.3: date of publication (Art. 21); sub-module 0.4: citation of date (Art. 22 and Art.

155 40.2.1); sub-module 0.5: electronic publication and generalities on publication (Art. 8.5, and

156 various recommendations related to publication); sub-module 0.6: publication as physical copies

157 (Art. 8.4); sub-module 0.7: General Recommendations (3-9); and sub-module 0.8: Ethics and

158 Recommendations (Code of Ethics and General Recommendations 10-12).

159 Module 1 contains 153 Nodes and 748 Edges, representing 11,09\% of the Code; it is the

160 fourth-largest module in the network (Figure 1). Module 1 mostly contains items from Chapter 2

161 (Number of words in scientific names; all items except Art. 4.1), a large part of Chapter 4

162 (Criteria of Availability; most of Art. 11 and parts of Arts. 10, 15, and 16), a large part of

163 Chapter 7 (Formation and treatment of names; mostly those parts that deal with the spellings of

164 specific names), the largest part of Chapter 10 (Species-group nominal taxa and their names),

165 and the part of Chapter 11 dealing with species-group names (Authorship; Art. 50.3.1, Rec. 51A,

166 Art. 51.3.3 and its example). This Module also contains a few individual items from Chapter 1

167 (Art. 1.3.4), Chapter 6 (Arts. 23.3.3, 23.3.4, and Example of Art. 24.1), Chapter 9 (Art. 42.1),

168 Chapter 12 (some examples and recommendations on specific names), Chapter 15 (Art. 67.5.3

169 and the Example of Art. 67.12.1), and Chapter 16 (Rec. 72C). The most important Glossary

170 terms included in this Module are species-group name, species group, name of species,

171 combination, specific name, binomen, subspecies, and other terms related to the species group.

172 Therefore, Module 1 deals primarily with names in the species group, their availability, as well 
173 as with general concepts like the binominal nomenclature. It should be noted that articles dealing

174 with types in the species groups are not included in this Module, but are mostly in the adjacent

175 Module 8 (see below). A secondary modularity analysis allows understanding better the

176 conceptual structure of Module 1. It consists of six sub-modules, which deal with the following

177 topics (Figure 2): sub-module 1.0: scientific names and binomina (most of Arts. 4-6, and other

178 related items); sub-module 1.1: species-group names (Art. 45, and other related items); sub-

179 module 1.2: availability of names of collective groups, ichnotaxa, divisions and other special

180 cases (Arts. 10.3, 10.4, 10.7); sub-module 1.3: availability requirements (most of Art. 11); sub-

181 module 1.4: availability of species-group names (Art. 11.9.3); sub-module 1.5: species-group

182 names and spellings (Arts. 31, 32 and other related items).

183 Module 2 contains 80 Nodes and 418 Edges, representing 5,8\% of the Code; it is the

184 second smallest module in the Network (Figure 1). Module 2 mostly contains items from

185 Chapter 4 (the majority of the information contained in the Articles in this chapter, exc. Art. 11

186 that is contained in other modules) and Chapter 11 (most of Art. 50, on authors). This Module

187 also contains some individual items from other Chapters, including Chapter 1 (Arts. 1.3.6, 1.3.7),

188 Chapter 5 (Rec. 22A.1, on the citation of date), Chapter 6 (Art. 23.3.7), Chapter 10 (Art. 45.5.1),

189 Chapter 12 (Art. 54.2), and Chapter 16 (Art. 72.5.1). The most important Glossary terms

190 included in this Module are: available name, plenary power, Articles, taxonomic information,

191 and unavailable name. Therefore, this Module deals primarily with the concept of availability of

192 names named before or after some special dates, but without the articles that deal with

193 publication (that are mostly included in Module 0). There is a significant overlap and contact

194 between Module 2 and 0 , which together contain most information on the availability of names.

195 A secondary modularity analysis allows understanding better the conceptual structure of Module 
196 197 198 199

200

201

202 203 204

205

206

207

208

209

210

211

212

213

214

215

216

217

218

2. It consists of five sub-modules, which deal with the following topics (Figure 2): sub-module 2.0: citation of authors (Rec. 22A.1, Art. 50.2); sub-module 2.1: availability before 1931 (Art. 12.2 and related items); sub-module 2.2: availability after 1930 (Art. 13 and related items); submodule 2.3: additional provisions on availability (the second half of Chapter 4 and related items); sub-module 2.4: additional provisions for the citation of authors (Arts. 50.1.1, 50.1.3). Module 3 contains 187 Nodes and 993 Edges, representing 13.56\% of the Code; it is the second largest Module in the network (Figure 1). Module 3 mostly contains items from Chapter 6 (Validity; most items of Arts. 23 and 24), Chapter 12 (Homonymy; entire Arts. 52, 56, and most of the other Articles of that Chapter), and several Articles from Chapter 17 (Commission; Arts 81.2, 81.2.1-4, 82, 82.1, 82.2). This Module also contains some individual items from other Chapters, including Chapter 4 (examples of Arts. 11.5.2 and 11.6.1), Chapter 7 (example of Art. 33.3.1), Chapter 8 (Arts. 38, 39, and examples of 40.1 and 40A), Chapter 9 (Art. 44.2), Chapter 10 (Arts. 44.2 and 47.2), Chapter 11 (Art. 50.6 and its example), Chapter 13 (Art. 61.2.1 and its example), Chapter 15 (Art. 67.1.2, Rec. 67B, and the example of Art. 67.8.1), and Chapter 16 (Art. 72.9). The most important Glossary terms included in this Module are: valid, species homonym, valid name, synonym, family homonym, genus homonym, Principle of Priority, and priority. This Module also contains the most important Article of the Code, which is Art. 23.1. Clearly, Module 3 deals with the validity of names, expanding also to the concepts of homonymy and synonymy which are tightly connected. A secondary modularity analysis allows understanding better the conceptual structure of Module 3. It consists of eight sub-modules, which deal with the following topics (Figure 2): sub-module 3.0: priority and homonymy of genus-group names (most of Art. 23, excl. provisions on family-group names and reversal of precedence, and Art. 56); sub-module 3.1: reversal of precedence (Art. 23.9-10, and related 
219 items); sub-module 3.2: precedence of simultaneously published names, spellings or acts and the

220 First Reviser (Art. 24 and related items); sub-module 3.3: homonymy in the family group and

221 their suppression (Arts. 38, 39, 54, 55, 81.2, and related items); sub-module 3.4: additional

222 provisions on validity, homonymy, synonymy (Arts. 23.3, 23.7, 23.12, 60.1-3, and related

223 items); sub-module 3.5: Principle of Homonymy (Art. 52 and some related items); sub-module

224 3.6: homonymy in family, genus, species, and variant spellings; and sub-module 3.7: secondary 225 homonyms (Arts. 57.3, 59, and related items).

226 Module 4 contains 82 Nodes and 351 Edges, representing 5.95\% of the Code; it is the third 227 smallest module of the network (Figure 1). Module 4 mostly contains the majority of the items 228 from Chapter 7. Exceptions include mostly Arts. 29 (Family-group names) and 30 (Gender of 229 genus-group names) which are included in Modules 6 and 5 respectively. Module 4 also contains 230 items from Chapter 4 (Arts. 11.9.1.1-4 on species-group names, Arts. 19.1-4 on spellings), 231 Chapter 6 (Art. 24.2.3 on original spellings), Chapter 8 (Art. 35.4 and the example of Art. 232 35.4.1), Chapter 9 (Art. 42.4), Chapter 11 (Arts. 50.4, 50.5, 51.3.1, on authorships), and Chapter 23312 (Art. 54.3 and 58.14, on spellings). The most important Glossary terms are: spelling, new 234 scientific name, incorrect subsequent spelling, incorrect original spelling, and original spelling. 235 Therefore, this Module contains most of the information on the formation of names and 236 spellings, focusing more on species-group names and excluding family- and genus-group names. 237 A secondary modularity analysis allows understanding better the conceptual structure of Module 238 4. It consists of five sub-modules, which deal with the following topics (Figure 2): sub-module 239 4.0: formation of species-group names (Arts. 11.9, 31.1, 58.14, and related items); sub-module 240 4.1: spellings (Arts. 32, 32, and related items); sub-module 4.2: inadvertent errors (Art. 32.5.1, 241 and related items); sub-module 4.3: emendations and their authors (Arts. 19.1-4, 32.2.2, 33.2.1- 
$2423,50.4,50.5,51.3 .1$ and related items); sub-module 4.4: formation of names (Arts. 25-34, and 243 related articles).

244 Module 5 contains 75 Nodes and 281 Edges, representing $5.44 \%$ of the Code; it is the 245 smallest module of the network (Figure 1). Module 5 mostly contains items from Chapter 7, and 246 especially those from Art. 30 on the Gender of genus-group names. It also contains many items

247 from Chapter 11, that is Articles dealing with the authorship of names and especially in 248 combination with genus-group names. Additionally, this Module contains some items from 249 Chapter 4 (example of Art. 11.3 and Rec. 11A), Chapter 5 (Art. 22 and Recs. 22A and 22A.3, 250 dealing with the citation of date and especially in combination), and Chapter 10 (Art. 48, on 251 change of generic assignment). The most important Glossary terms are: author, Latin, gender, 252 gender ending, Greek, compound, latinize, gender agreement, and new combination. Therefore, 253 this module deals also with the formation of names, focusing however in the genus-group name, 254 combination and gender agreement in particular. A secondary modularity analysis allows 255 understanding better the conceptual structure of Module 5. It consists of five sub-modules, which 256 deal with the following topics (Figure 2): sub-module 5.0: gender of names formed from Latin or 257 Greek words (Art. 30.1); sub-module 5.1: gender of names not formed from Latin or Greek 258 words (Art. 30.2); sub-module 5.2: gender agreement (Arts. 31.2, 34.2, 48); sub-module 5.3: 259 citation of author and date, related mostly to combinations (Arts. 22, 50.3, 51); sub-module 5.4: 260 examples of the gender of names formed from Latin or Greek words (examples of relevant 261 articles). Module 6 contains 138 Nodes and 703 Edges, representing 10.01\% of the Code; it is the 263 sixth-largest module in the network (Figure 1). Module 6 mostly contains items from Chapter 7, 264 and especially those dealing with the formation of family-group names. It also contains several 
265 items from Chapter 4 (articles and examples on family-group names), most items from Chapter 8

266 (all on the family-group names), and Chapter 14 (all on name-bearing types of family-group

267 names). Additionally, this module contains some individual items from Chapter 6 (priority on

268 family-group names), Chapter 9 (Principle of Coordination in the genus-group), Chapter 10

269 (Principle of Coordination in the species-group), Chapter 12 (on homonymy in the family-

270 group), Chapter 13 (types on nominotypical taxa), Chapter 15 (on type species), Chapter 16 (on

271 type specimens), and Chapter 17 (on the use of plenary power related to rank). The most

272 important Glossary terms included in this module are: taxon, family-group, family-group name,

273 rank, scientific name, suffix, type genus, stem, family name, superfamily, Principle of

274 Coordination, subfamily name, and superfamily name. Therefore, this Module mostly deals with

275 the formation and application of family-group names, and its name-bearing types. This Module

276 also contains the basic information on the Principle of Coordination and, thus, shows a strong

277 overlap with Modules 7 and 8 that deals with the genus- and species-group in particular. A

278 secondary modularity analysis allows understanding better the conceptual structure of Module 6.

279 It consists of six sub-modules, which deal with the following topics (Figure 2): sub-module 6.0:

280 availability of family-group names (Art. 11.7.1, 64); sub-module 6.1: Principle of Coordination

281 and some provisions on name-bearing types (Arts. 34.1, 36, 37, 43, 44, 46, 47, 61.2, 63.1, 67.1.1,

282 72.8); sub-module 6.2: formation of family-group names (Arts. 29.2, 29.4.1, 32.5.3, 35.2, 53.1);

283 sub-module 6.3: formation of family-group names, stem and spellings (Arts. 23.1.2, 23.5, 29.1,

$28429.3-6,33.2 .3 .1,33.3 .1,35.4 .1-2,55.3 .1$ and related items); sub-module 6.4: validity and

285 synonymy of family-group names (Arts. 23.1.1, 35.3, 35.5, 40.1, 40.2, 41, 63, 65); sub-module

286 6.5: miscellaneous information on family-group names, examples and definitions (examples of

287 relevant articles and Glossary terms). 

fifth largest module in the network (Figure 1). This Module contains the great majority of items included in Chapter 15 (types in the genus group) and several items from various Articles of

291 Chapter 4 (all dealing with the genus-group names) and Chapter 9 (Genus-group). Additionally, 292 this Module contains some individual items of Chapter 1 (Art. 1.2.1, on collective groups), 293 Chapter 5 (examples on citation of dates related to genus-group names), Chapter 6 (Art. 23.7.2, 294 priority in genus/collective group), Chapter 10 (Art. 49), Chapter 11 (some items on type 295 species), Chapter 12 (some items on homonymy), and Chapter 13 (Art. 61.4 and its example, on 296 nominal subgenus). The most important Glossary terms included in this module are: species, 297 establish, genus group, genus-group name, type species, generic name, deem, and subgenus. 298 Therefore, this module deals mainly with genus-group names and their application, as well as the 299 type species. A secondary modularity analysis allows understanding better the conceptual 300 structure of Module 7. It consists of six sub-modules, which deal with the following topics 301 (Figure 2): sub-module 7.0: type species (Art. 67); sub-module 7.1: availability of genus-group 302 names and type fixation (Arts. 13.3, 16.3, 42, 66, 67.4.1, 67.12.1, 68, 69.4, 70.2, 70.3); sub303 module 7.2: type species designations (Arts. 67.2, 67.3.2, 67.8.1, 69.1, 69.2); sub-module 7.3: subsequent designation recommendations (recommendations of Art. 69); sub-module 7.4: types in the genus group (several items of Arts. 67, 68, 69, 70 and related items); sub-module 7.5: type 306 fixation, additional provisions (Arts. 67.2.1, 67.13, 68.2, 68.6, 70.3, 70.4 and related items). Module 8 contains 200 Nodes and 978 Edges, representing $14.5 \%$ of the Code; it is the 308 largest module in the network (Figure 1). This Module contains the great majority of items 309 included in Chapter 16 (Types in the species group), and several items from Chapter 4 (most of 310 Art. 16 on names published after 1999) and Chapter 13 (most of Art. 61 on the Principle of 
311 Typification). Additionally, this Module contains some individual items of Chapter 3 (Rec. 8B), 312 Chapter 7 (Rec. 25C), Chapter 12 (Art. 45.3), and Chapter 17 (Arts. 79.1.3 and 79.5.2). The most

313 important Glossary terms included in this Module are: Recommendation, name-bearing type,

314 specimen, nominal taxon, lectotype, holotype, syntype, neotype, description, type series, nomen

315 novum, and taxonomy. Clearly, this Module deals with typification and particularly with types

316 on the species group. A secondary modularity analysis allows understanding better the

317 conceptual structure of Module 8. It consists of eight sub-modules, which deal with the following

318 topics (Figure 2): sub-module 8.0: Principle of Typification (Arts. 61.1, 61.3, and related terms);

319 sub-module 8.1: types in the species group and neotypes (Art. 71, 72, 75, and related terms); sub-

320 module 8.2: availability-related provisions on name-bearing types (Arts. 16.1, 16.2, 16.4, and

321 related items); sub-module 8.3: recommendations on type specimens and type localities

322 (recommendations of Arts. 16, 25, 72, 73, 74, 76); sub-module 8.4: type series (Art. 72.4 and

323 related items); sub-module 8.5: holotypes, hapantotypes (Arts. 72.5, 72.6, 73.1, 73.3); sub-

324 module 8.6: syntypes, lectotypes (Arts. 73.2, 74, and related items); sub-module 8.7: neotypes

325 and type locality (Arts. 75.3, 76.1-3).

326 Finally, Module 9 contains 136 Nodes and 567 Edges, representing 9.86\% of the Code; it

327 is the fourth smallest module in the network (Figure 1). This Module contains the great majority

328 of items included in Chapter 17 (Commission), and all items of Chapter 18 (Regulations). Also,

329 this Module contains individual items of Chapter 4 (Art. 10.6), Chapter 11 (example of 51F),

330 Chapter 12 (Arts. 57.2.2-3, 59.3.1), and Chapter 16 (example of Art. 75.6). The most important

331 Glossary items included in this Module are: Commission, Code, provisions, adopt, Opinion,

332 ruling of the Commission, List of Available Names, Part of the List of Available Names,

333 proposal, and index. Therefore, this module clearly contains almost all the information on the 
334 Commission and its regulations. A secondary modularity analysis allows understanding better

335 the conceptual structure of Module 9. It consists of seven sub-modules, which deal with the

336 following topics (Figure 2): sub-module 9.0: Relations of the Commission (Art. 77, and related

337 items); sub-module 9.1: powers and duties (Art. 78); sub-module 9.2: List of Available Names

338 (Art. 79, and related items); sub-module 9.3: List of Available Names requirements (Art. 79.2);

339 sub-module 9.4: Status of works, names and nomenclatural acts in Official Lists (Art. 80.6);

340 sub-module 9.5: Opinions and Corrections (Arts. 80.2-5, 80.7, 80.9, and related items); sub-

341 article 9.6: regulations governing the Code (Arts. 85-89).

342

343 Parcellation Index. On one hand, the Code has a given, de facto, structure: it is the current

344 structure of the Code, with its Articles that are grouped in Chapters, all placed in their order. On

345 the other hand, the Code has another, conceptual, structure: this is the structure that is revealed

346 by the modularity analysis and is based on the conceptual connections between the various parts

347 of the Code. The PI allows calculating the agreement between these two structures (Table 1 and

348 Figure 3), and highlight how the presentation of the Code could be improved. When these two

349 structures agree, the PI is low or zero. For example, Chapter 14 is included entirely in Module 6,

350 and Chapter 18 is included entirely in Module 9. On the other hand, a Chapter that is spread

351 across many Modules, with various items in each Module, will be highly parcellated, and its PI

352 will be high, approaching 1. For example, the contains of Chapter 4 are placed in all 9 recovered

353 Modules, and its parcellation index is 0.787 (Table 1). This new analysis reveals that six

354 Chapters of the Code have excellent structure and composition (Figure 3): Chapters 2, 3, 14, 15,

355 16, and 18. Another six Chapters have good structure and composition: Chapters 1, 5, 6, 8, 12 ,

356 and 17. Therefore, two-thirds of the Code have actually a quite good conceptual structure that 
357 agrees quite well with the de facto structure. However, the remaining six chapters $(4,7,9,10,11$,

358 and 13) are in the "red zone", with two of them (4 and 11) crossing the 0.750 boundary. If we

359 want to improve the current structure of the Code, we should focus on the placement and

360 composition of these six problematic chapters.

361 Chapter 4 deals with availability and is arguably one of the most important Chapters of the

362 Code. It contains many Articles (Arts. 10-20), matched only by Chapter 7 in number of Articles

363 - it has, however, more individual items (134) toped only by Chapter 16 with 140 items. More

364 importantly, the included Articles in this Chapter are quite heterogeneous and for three good

365 reasons. First, Art. 10 contains few items, but most of these are related with parts contained in

366 the first Chapters of the Code. Second, Chapter 4 includes items on the availability on names in

367 different groups: family-group, genus-group, and species-group (Art. 11); these items are

368 conceptually closer to the recovered modules dealing with these groups. Finally, this Chapter

369 contains separate Articles that deal with the availability of names published in different times

370 (1930, 1960, 1999), all of which are conceptually closer with other parts of the Code. Chapter 7

371 deals with the Formation and treatment of names, and suffers as well from one of the problems

372 of Chapter 4: Art. 29 deals with family-group names, Art. 30 with genus-group names, and Art.

37331 with species-group names. However, only the latter (Art. 31) appears to be highly parcellated.

374 Also, the remaining Articles on spellings and emendations (Arts. 32-34) are also highly

375 parcellated, as they also deal with names for taxa in different ranks. Chapter 13 deals with the

376 Principle of Typification and suffers again from the same problem: its items are conceptually

377 closer to modules dealing with types in different groups. The analysis of the PI of these three

378 chapters reveals the first important problem of the structure of the Code: information dealing

379 with names in the family, genus, and species group are placed in the same Article or Chapter, 
380 although they are conceptually different. A possible improvement, therefore, would be to create

381 chapters the contain information for the same group only.

382 Chapter 9 deals with genus-group nominal taxa and their names. Although it contains three

383 different Articles (Arts. 42-44), this is actually a quite short Chapter that contains only 15 items.

384 Most of these are placed in Module 6 and 7, but some solitary items are placed elsewhere.

385 Therefore the high PI is an artefact of the low number of items of this chapter, and its structure is 386 not considered to be a significant problem herein.

387 Chapter 10 deals with the species group. Most of its items are placed in Module 1, however 388 items from Arts. 46 and 47 that deal with Coordination are placed elsewhere. The parcellation 389 analysis of these two chapters reveals another problem: the Principle of Coordination is an 390 important concept that forms part of a different conceptual Module and it would be perhaps 391 better to group all these articles together.

392 Finally, Chapter 11 deals with authorship, and is a highly parcellated chapter that contains 393 heterogeneous information. For example, Art. 50 contains items that deal with many different 394 concepts: authorship (Arts. 50.1-2), rank (Art. 50.3), combination (Art. 50.3), emendations 395 (Arts. 50.4-5), date of publication (Art. 50.6), and synonymy (Art. 50.7). All these items are 396 better grouped and placed elsewhere, in their conceptual modules, and that is why this Article is 397 the one with the highest PI in the Code.

398

399 Contact between the Modules. As we have seen, the contact between the various modules is 400 quite extensive. All the modules together sum up to 8,928 Edges, meaning that 2,348 (a bit more 401 than $20 \%$ ) of the total 11,276 Edges of the network are responsible for connecting the modules to 402 each other. The NETICON allows identifying exactly which are these Edges connecting Nodes 
403 between the modules (Table 2), and could be used for the fine-tuning of the structure of the

404 Code. The most extensive contact zones are found between module 1 and module 7 (availability

405 of species-group names and types in the genus group; 233 connecting edges), module 1 and

406 module 3 (availability of species-group names and validity, homonymy, synonymy; 209

407 connecting edges), and module 0 and module 9 (introduction, generalities, publication and

408 Commission, regulations; 197 connecting edges). Important contact zones are found between

409 module 3 (validity, homonymy, synonymy) with module 6 (formation of names in the family

410 group; 183 interconnecting edges), module 7 (types in the genus group; 186 interconnecting

411 edges), and module 9 (Commission, regulations; 121 interconnecting edges), and the main

412 availability module (module 1) with nearly all other modules. The least extensive contact

413 between modules is found between module 4 and module 8 (formation of names in the species

414 group and types in the species group; 10 interconnecting edges), and most other modules (exc. 0

415 and 3) with the module related to the Commission and regulations (module 9).

416

\section{Discussion}

418 The modularity analysis performed herein helps formulating recommendations for

419 developing a more "reader-friendly" version of the Code, a version that will reflect more the

420 conceptual clusters within the Code and less the historical, de facto, structure. This new version

421 should be complementary to the current one, and the current numbering should be used as

422 reference only and not for organization purposes. Having a version that is better organized could

423 certainly improve the applicability of the Code, especially by those users that are not so

424 experienced with all the Rules. Also, a better organized version would help a lot in teaching the

425 Code to students and beginners. 

created by the placement of provisions dealing with the different and important concepts of the

428 Code (publication, availability, typification, validity) for each group (i.e., family, genus, species 429 group) in different chapters organized per concept. And this is further complicated if we add a 430 temporal element, as there are in many cases different provisions for taxa named before or after 431 certain dates. It seems that a different organization, grouping together all the provisions dealing 432 with taxa and their names in the same rank, is desirable to improve the structure of the Code. The only concept that appears, currently, to override this organization is Homonymy, whose relevant provisions are mostly found in the same conceptual module.

Once this conflict is solved, the next step would be to resolve the second point of conflict, that is the different provisions depending on several Important Dates in the history of the Code.

437 For the moment it seems that all relevant provisions for most of the specific dates form different 438 sub-modules within the larger ones, so a new modularity analysis could help to prove whether 439 more changes, and which, are necessary or not.

Also, the modularity analysis suggests that it might be desirable to merge Chapter 3 with 441 Chapter 5. As we have seen, the items of Chapter 5 (Arts. 21 and 22) that deal with the date of 442 publication and its form of citation, are conceptually included in the Module that also includes 443 Chapters 1 and 3. Moving Chapter 5 before Chapter 4, or even include its Articles in Chapter 3, 444 would agree more with the conceptual structure of the Code. Additionally, individual articles that 445 are spread in other parts of the Code but also deal with the date of publication (e.g., Art. 40.2.1) 446 could also merit inclusion. Chapter 11, on authorship, is highly parcellated, and it is better if it is 447 dissolved and its articles placed in their corresponding modules. 
Once the main modularity conflicts are resolved, another issue needs to be further

449

450

451

452

453

454

455

456

457

458

459

460

461

462

463

464

465

466

467

468

469

470

investigated: the order of the various concepts in the continuous text. Currently, the order of

appearance is from the higher rank to the lower one (i.e., family, genus, species). However, most

of the modularity searches, following the connections within the Code, usually followed the

inverse path: species, genus, family. This is something that needs to be further investigated, but it

could perhaps provide an even better reader experience. Finally, in all runs, the beginning

(Chapter 1) and the end of the Code (Chapters 18, 19, and Appendices) are always close together

with an important contact zone. It appears that this general information should be grouped together, probably in the beginning of the Code.

Based on the modularity analysis, it seems that a reasonable proposal for the reader-

friendly version of the Code would be the following: First Part: Introduction and Generalities,

General Recommendations, Code of Ethics, Commission and Regulations; Second Part: General information on Publication, Availability, and Validity; Main Part: Species group, Genus group,

Family Group. As all these conceptual modules are not independent but rather present extensive

contact zones, it might be desirable that certain information from these contact zones should be

repeated in various parts of the Code to provide both conceptual closure of the various concepts

as well as connection between the different concepts.

A final point to be made refers to the Glossary. As expected, the Glossary is highly parcellated $(0,890)$, and its terms are included in all modules and distributed quite evenly across modules. Currently, the Glossary is placed in the end of the Code, and both in print and online versions it requires extra effort to check the Glossary terms. In many cases, as exemplified in detail in my earlier paper (Vlachos, 2019) the understanding of the Glossary terms is key to understand and apply the Code. So, a final change to greatly improve the structure and 
471 readability of the Code would be to include all the relevant Glossary terms in the same printed or

472 online page that they appear, perhaps in the form of footnotes or hove-over windows

473 respectively, or any other technical solution that might fit better to that purpose.

474 The modularity analysis could help in providing a new visual representation and structure

475 of the Code (Figure 4). Each sub-module is conceptualized as a different "nomenclatural

476 element", and using their connections inside and outside their larger module, we can place them

477 in a non-overlapping two-dimensional way, forming a table (Figure 4). In this diagram, the basic

478 Principles have, just like in the network, a central position. From left-to-right we go from more

479 general elements to more specific ones, whereas the mid-and-right part of the diagram is divided

480 in the four important structural items: availability, validity, formation of names, and typification.

481 The right part of the diagram is divided vertically and successively from left-to-right in the

482 species-, genus-, and family-group. Some modules are entirely included in one of these five

483 structural areas, whereas others not. The various network metrics of each element could help in

484 understanding the importance of each element (see Vlachos 2019 for detailed explanation of the

485 various metrics). The number of items and average weighted degree tell the reader the amount

486 and importance of the information included in this element. Elements with high density and high

487 clustering are "heavy" elements, suggesting that these elements could stand and be understood

488 on its own. Elements with low density and low clustering are more "unstable", meaning that

489 need to be read and understood in combination with other surrounding elements. This diagram

490 could assist in using and teaching the Code, as well as to provide a starting point for the

491 development of a virtual application.

492

493 Acknowledgments 
494 I would like to thank the Thomas Pape and the International Commission on Zoological

495 Nomenclature for granting me permission to use the text of the Code for the analyses herein.

496

\section{References}

498 Bastian M, Heymann S, Jacomy M. 2009. Gephi: an open source software for exploring and 499 manipulating networks. Proceedings of the third International ICWSM Conference 8:361$500 \quad 362$.

501 Blondel VD, Guillaume JL, Lambiotte R, Lefebvre E. 2008. Fast unfolding of communities in 502 large networks. Journal of statistical mechanics: theory and experiment 2008(10):P10008.

503 Calatayud J, Bernardo-Madrid R, Neuman M, Rojas A, Rosvall M. 2019. Exploring the solution 504 landscape enables more reliable network community detection. Physical Review E $505 \quad 100(5): 052308$.

506 Esteve-Altava B, Pierce SE, Molnar JL, Johnston P, Diogo R, Hutchinson JR. 2019.

507 Evolutionary parallelisms of pectoral and pelvic network-anatomy from fins to limbs. Science 508 Advances 5(5):eaau7459.

509 ICZN [International Commission on Zoological Nomenclature]. 1999. International code of

510 zoological nomenclature. 'Fourth edition'. London: (International Trust for zoological $511 \quad$ Nomenclature).

512 Vlachos E. 2019. Introducing a new tool to navigate, understand and use International Codes of $513 \quad$ Nomenclature. PeerJ 7:e8127. 


\section{Table 1 (on next page)}

Distribution of the items of each Chapter of the Code across modules.

The various text parts of the Chapters (Ch.) of the Code are included in different conceptual modules, as revealed by the modularity analysis. "Other" refers to texts not included in the Chapters, i.e., the Glossary and the Appendices. Their Parcellation Index (PI) is indicated as well. 


\section{Table 1:}

\section{Distribution of the items of each Chapter of the Code across modules.}

3 The various text parts of the Chapters (Ch.) of the Code are included in different conceptual

4 modules, as revealed by the modularity analysis. "Other" refers to texts not included in the

5 Chapters, i.e., the Glossary and the Appendices. Their Parcellation Index (PI) is indicated as

6 well.

\begin{tabular}{|c|c|c|c|c|c|c|c|c|c|c|c|c|}
\hline \multicolumn{10}{|c|}{ Modules } \\
\hline Ch. & $\mathbf{0}$ & $\mathbf{1}$ & $\mathbf{2}$ & $\mathbf{3}$ & $\mathbf{4}$ & $\mathbf{5}$ & $\mathbf{6}$ & $\mathbf{7}$ & $\mathbf{8}$ & $\mathbf{9}$ & $\mathbf{N}$ & PI \\
\hline $\mathbf{1}$ & 19 & 1 & 2 & - & - & - & - & 1 & - & - & $\mathbf{2 3}$ & 0,306 \\
\hline $\mathbf{2}$ & 0 & 14 & - & - & 1 & - & - & - & - & - & $\mathbf{1 5}$ & 0,124 \\
\hline $\mathbf{3}$ & 51 & - & - & - & - & - & - & - & 1 & - & $\mathbf{5 2}$ & 0,038 \\
\hline $\mathbf{4}$ & 6 & 41 & 41 & 2 & 9 & 2 & 11 & 11 & 10 & 1 & $\mathbf{1 3 4}$ & 0,787 \\
\hline $\mathbf{5}$ & 27 & - & 1 & - & - & 3 & - & 2 & - & - & $\mathbf{3 3}$ & 0,318 \\
\hline $\mathbf{6}$ & 1 & 3 & 1 & 47 & 1 & - & 4 & 1 & - & - & $\mathbf{5 8}$ & 0,335 \\
\hline $\mathbf{7}$ & - & 25 & - & 1 & 39 & 35 & 27 & - & 1 & - & $\mathbf{1 2 8}$ & 0,75 \\
\hline $\mathbf{8}$ & 2 & - & - & 4 & 2 & - & 21 & - & - & - & $\mathbf{2 9}$ & 0,447 \\
\hline $\mathbf{9}$ & - & 1 & - & 1 & 1 & - & 5 & 7 & - & - & $\mathbf{1 5}$ & 0,658 \\
\hline $\mathbf{1 0}$ & - & 16 & 1 & 2 & - & 1 & 5 & 2 & 1 & - & $\mathbf{2 8}$ & 0,628 \\
\hline $\mathbf{1 1}$ & 3 & 4 & 10 & 2 & 3 & 13 & - & 4 & - & 1 & $\mathbf{4 0}$ & 0,798 \\
\hline $\mathbf{1 2}$ & 1 & 2 & 1 & 72 & 2 & - & 5 & 2 & 1 & 3 & $\mathbf{8 9}$ & 0,339 \\
\hline $\mathbf{1 3}$ & - & - & - & 2 & - & - & 2 & 2 & 10 & - & $\mathbf{1 6}$ & 0,563 \\
\hline $\mathbf{1 4}$ & - & - & - & - & - & - & 11 & - & - & - & $\mathbf{1 1}$ & 0 \\
\hline $\mathbf{1 5}$ & - & 2 & - & 3 & - & - & 1 & 85 & - & - & $\mathbf{9 1}$ & 0,126 \\
\hline $\mathbf{1 6}$ & - & 1 & 1 & 1 & - & - & 1 & - & 135 & 1 & $\mathbf{1 4 0}$ & 0,07 \\
\hline $\mathbf{1 7}$ & 1 & - & - & 9 & - & - & 2 & - & 2 & 80 & $\mathbf{9 4}$ & 0,266 \\
\hline $\mathbf{1 8}$ & - & - & - & - & - & - & - & - & - & 16 & $\mathbf{1 6}$ & 0 \\
\hline Other & 70 & 43 & 22 & 41 & 24 & 21 & 43 & 30 & 39 & 34 & $\mathbf{3 6 7}$ & 0,306 \\
\hline $\mathbf{N}$ & $\mathbf{1 8 1}$ & $\mathbf{1 5 3}$ & $\mathbf{8 0}$ & $\mathbf{1 8 7}$ & $\mathbf{8 2}$ & $\mathbf{7 5}$ & $\mathbf{1 3 8}$ & $\mathbf{1 4 7}$ & $\mathbf{2 0 0}$ & $\mathbf{1 3 6}$ & $\mathbf{1 3 7 9}$ & $\mathbf{0 , 8 8 9}$ \\
\hline
\end{tabular}

7 
Table 2 (on next page)

Contact between the various modules of the Code.

The number of edges or connections between the various recovered modules of the Code. 


\section{Table 2:}

\section{Contact between the various modules of the Code.}

3 The number of edges or connections between the various recovered modules of the Code.

\begin{tabular}{|r|r|r|r|r|r|r|r|r|r|r|}
\hline Modules & 0 & 1 & 2 & 3 & 4 & 5 & 6 & 7 & 8 & 9 \\
\hline 0 & - & 132 & 164 & 122 & 45 & 55 & 74 & 79 & 138 & 197 \\
\hline 1 & 132 & - & 140 & 209 & 105 & 127 & 127 & 233 & 163 & 45 \\
\hline 2 & 164 & 140 & - & 105 & 62 & 45 & 93 & 123 & 107 & 85 \\
\hline 3 & 122 & 209 & 105 & - & 68 & 29 & 183 & 186 & 94 & 123 \\
\hline 4 & 45 & 105 & 62 & 68 & - & 54 & 96 & 36 & 10 & 21 \\
\hline 5 & 55 & 127 & 45 & 29 & 54 & - & 63 & 74 & 66 & 18 \\
\hline 6 & 74 & 127 & 93 & 183 & 96 & 63 & - & 146 & 129 & 38 \\
\hline 7 & 79 & 233 & 123 & 186 & 36 & 74 & 146 & - & 147 & 41 \\
\hline 8 & 138 & 163 & 107 & 94 & 10 & 66 & 129 & 147 & - & 67 \\
\hline 9 & 197 & 45 & 85 & 123 & 21 & 18 & 38 & 41 & 67 & - \\
\hline
\end{tabular}

4 


\section{Figure 1}

The main modules of the Code

The 10 different conceptual modules of the International Code of Zoological Nomenclature based on the modularity analysis of the network of the Code (Neticon zoologicon). The modules are depicted in a strong gravity Force Atlas 2 layout. The most important nodes are highlighted: AUT, author; ART, Article; AVN, available name; COD, Code; COM, Commission; FAN, family name; GEN, genus; PUB, Publication; REC, recommendation; SP, species; SPN, species name; TAX, taxon; TSP, type species; TY, type; VA, valid name. 


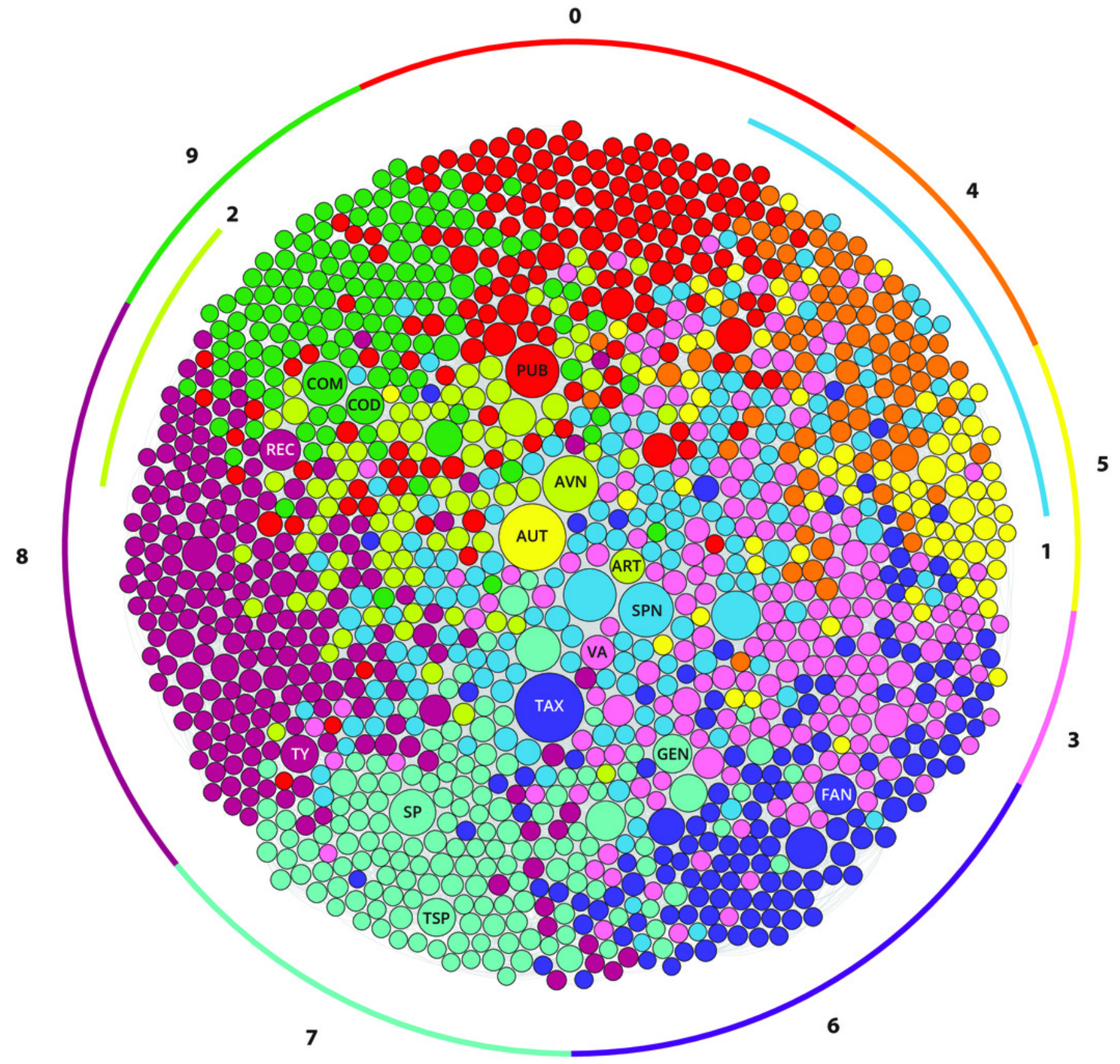


Figure 2

The composition of the different modules of the Code

Each of the 10 main modules of the Code (see Fig. 1) deals with important concepts, and contains several smaller sub-modules that group together the provisions on various smaller conceptual elements. The Code can be divided into 65 different conceptual elements, grouped into 10 larger ones, indicating the conceptual structure of the Code. 


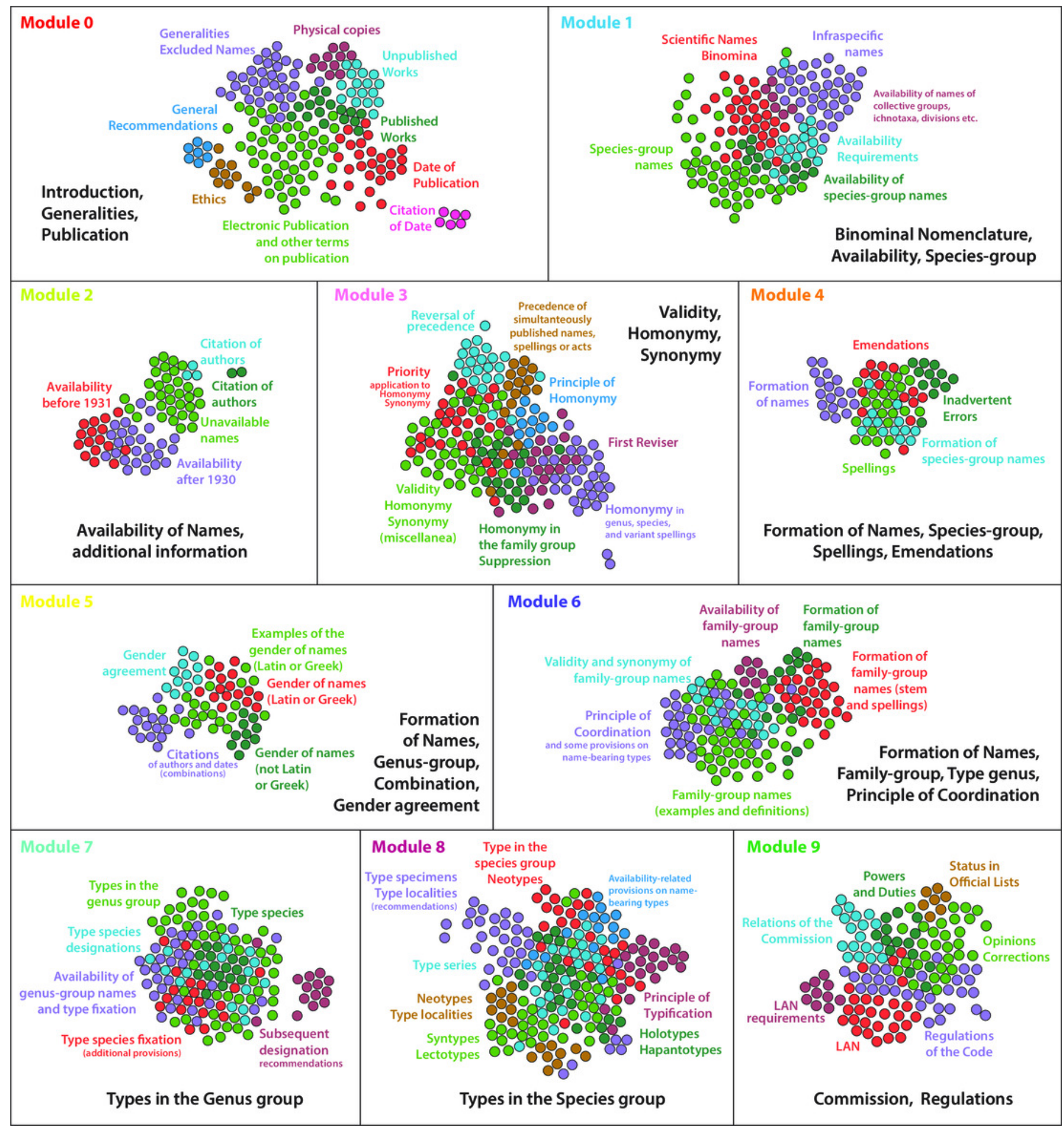




\section{Figure 3}

Evaluation of the structure of the various Chapters of the Code

Based on the recovered modules, the structure of each Chapter has been evaluated based on a parcelation analysis. Two-thirds of the Code appear to be included in Chapters with excellent or Code structure, whereas six chapters are considered to have problematic or bad structure. The most important problem is the inclusion of provisions dealing with names and taxa of different rank (species, genus, family) in the same Chapter.

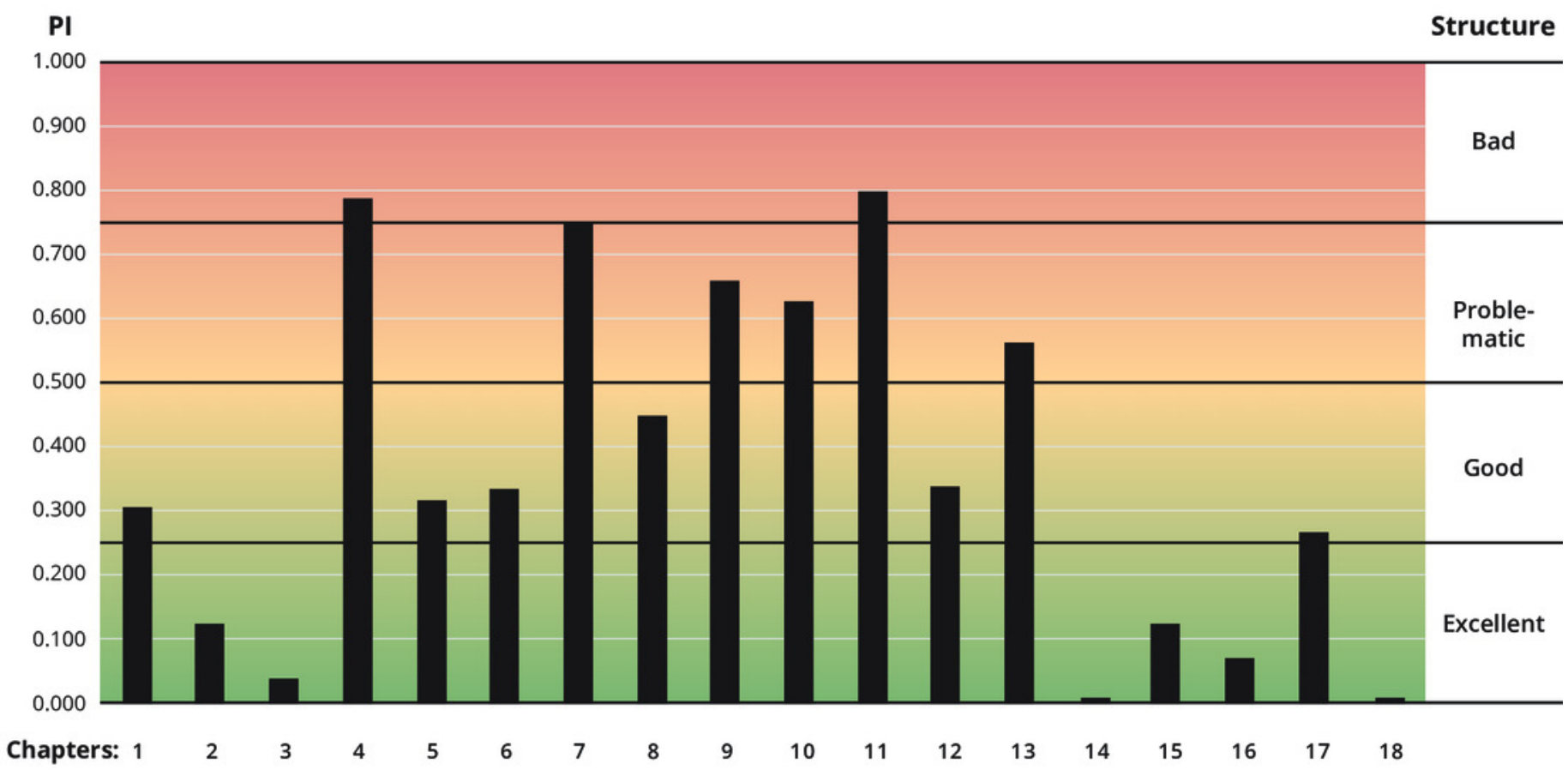




\section{Figure 4}

\section{The Periodic Table of Nomenclatural Elements}

The modularity analysis of the network of the Code allows dividing the Code into 65 different conceptual elements and place them in a non-overlapping, two-dimensional way. Each element includes its four main metrics. The most important elements have high number of items and high degree. "Heavy" elements are those with high density and clustering, and usually stand and be understood on their own, where "unstable" elements with low density and clustering need to be understood together with their surrounding elements. 


\section{The Periodic Table of Nomenclatural Elements}

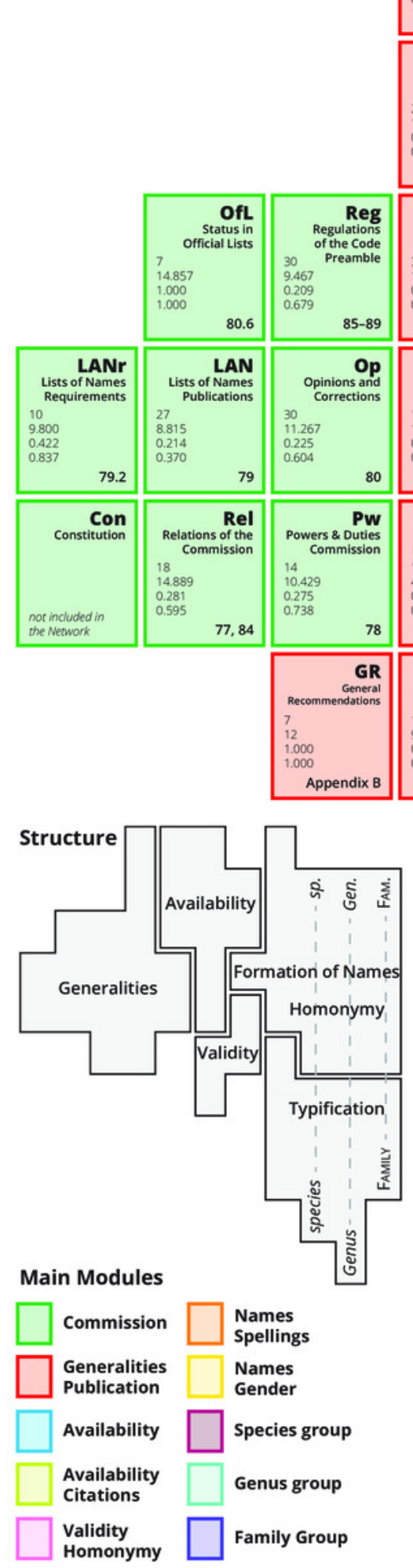

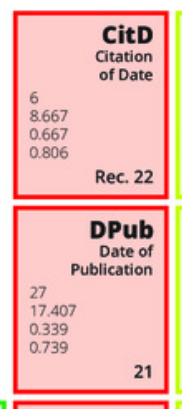
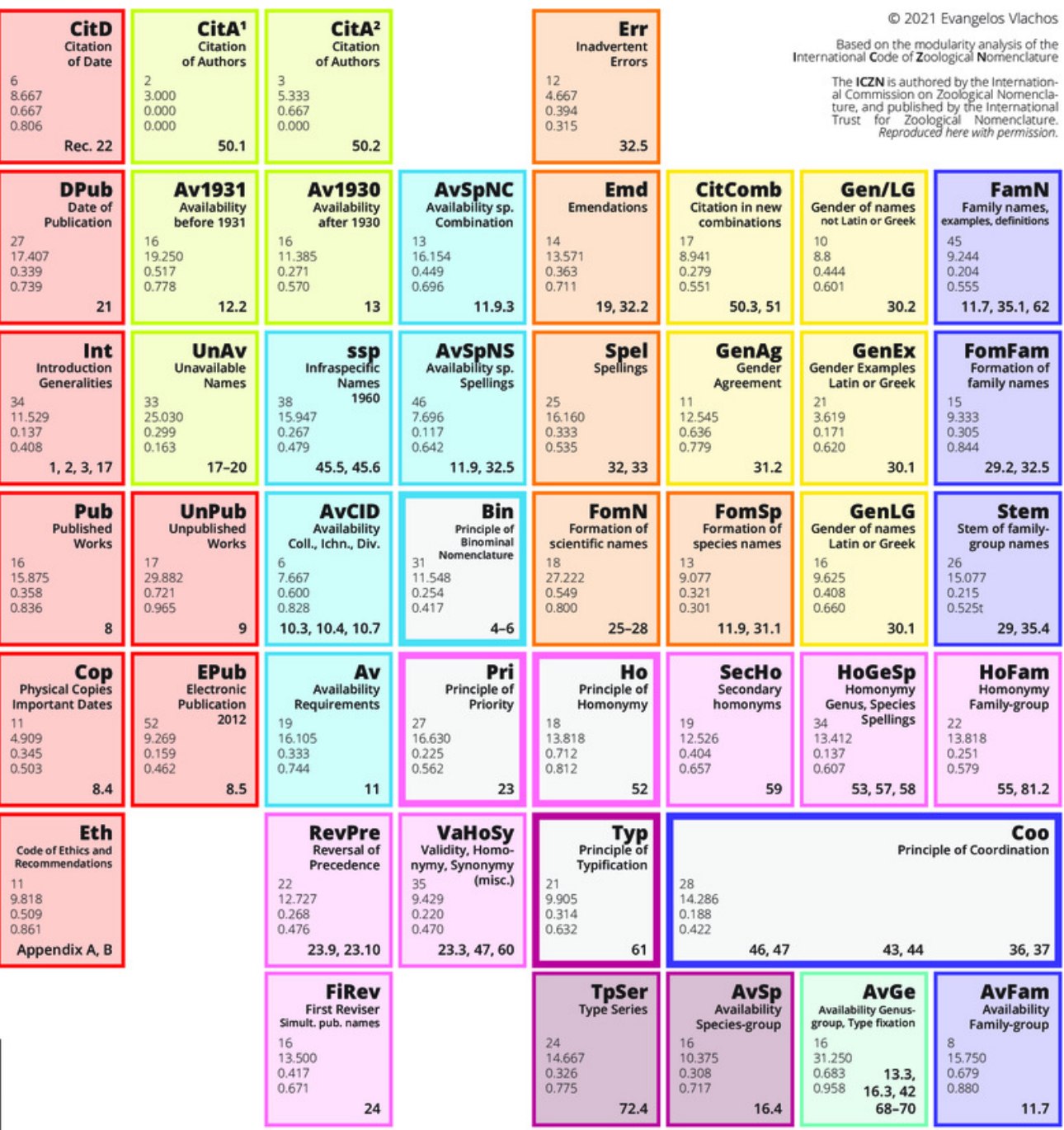

ture, and published by the International
Trust for Zoological Nomenclature.
Reproduced here with permission
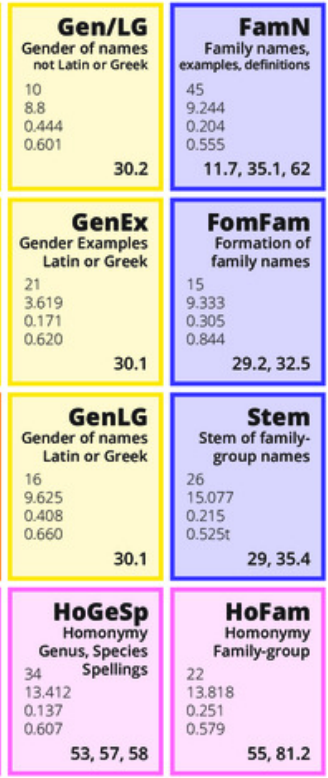

Principle of Coordination

\begin{tabular}{|c|c|c|}
\hline 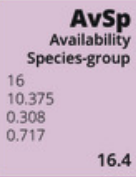 & 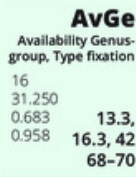 & $\begin{array}{l}\begin{array}{r}\text { AvFam } \\
\text { Availability } \\
\text { Family-group }\end{array} \\
8 \\
15.750 \\
0.679 \\
0.880\end{array}$ \\
\hline $\begin{array}{rr}\begin{array}{r}\text { HolTp } \\
\text { Holotypes } \\
\text { Hapantotypes }\end{array} \\
23 & \\
8.870 & \\
0.198 & \\
0.459 & \mathbf{7 2 . 5} \\
& \mathbf{7 3 . 1}, \mathbf{7 3 . 3}\end{array}$ & $\begin{array}{r}\text { TySp } \\
\text { Type Species }\end{array}$ & 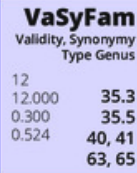 \\
\hline 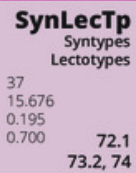 & 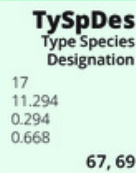 & 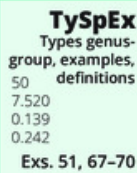 \\
\hline
\end{tabular}

Colors indicate the major conceptual Modules
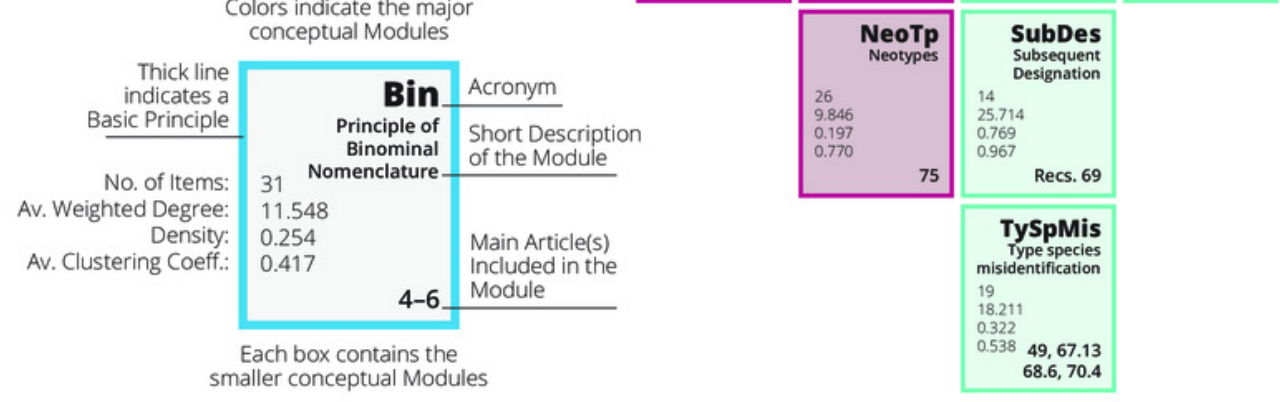